\title{
Chronic Intermittent Hypobaric Hypoxia (4600 M) Attenuates Pulmonary Vasodilation Induced by Acetylcholine or Sodium Nitroprusside
}

\author{
Fernando A. Moraga, Giselle Miranda, Vasthi López, Carmen Vallejos, and Daniel Silva
}

\begin{abstract}
Moraga, Fernando A., Giselle Miranda, Vasthi López, Carmen Vallejos, and Daniel Silva. Chronic intermittent hypobaric hypoxia $(4600 \mathrm{M})$ attenuates pulmonary vasodilation induced by acetylcholine or sodium nitroprusside. High Alt Med Biol. 19:149-155, 2018.

Background: Previous studies performed in rats exposed to chronic intermittent hypobaric hypoxia (CIHH), at a simulated altitude of $4600 \mathrm{~m}$, showed reduced nitric oxide (NO) production, increased arginase activity, and increased oxidative stress. However, studies on vascular function are scarce. Our aim was to measure plasma nitrate and nitrite (NOx) concentration and study pulmonary vascular function in rats exposed to $\mathrm{CIHH}$ in the presence of potassium chloride $(\mathrm{KCl})$, acetylcholine (Ach), and sodium nitroprusside (SNP).

Methods: Thirty male Wistar rats were divided into two groups: A control group (normoxia $(\mathrm{N}), n=10)$ and a $\mathrm{CIHH}$ group $(2 \mathrm{~N} \times 2 \mathrm{H} \times 30$ days, $n=20)$. $\mathrm{CIHH}$ exposure was performed in a hypobaric chamber at 428 Torr $(4600 \mathrm{~m})$. Noninvasive systolic blood pressure (SBP), heart rate, and body weight (BW) were measured. Blood samples were obtained to measure NOx levels and hematocrit (Hct). CIHH animals that gained BW and presented a Hct $<20 \%$ and maintained SBP were classified as tolerant, and animals that lost $>30 \%$ of their BW, increased Hct and SBP $>20 \%$ were classified as intolerant. Animals were sacrificed and small pulmonary arteries (SPA) were obtained to perform concentration-response curves to $\mathrm{KCl}$, Ach, and SNP.

Results and Conclusions: Intolerant rats (30\%) had decreased NOx levels. SPA had a larger vasocontraction response to $\mathrm{KCl}$ and a lower dilation response to SNP in the SPA compared to tolerant and control animals. In addition, SPA had a lower dilatation response to Ach compared with the control. Together, these results show that $\mathrm{CIHH}$ alters endothelium-dependent vasodilation.
\end{abstract}

Keywords: acetylcholine; chronic intermittent hypobaric hypoxia; hematocrit; NO; pulmonary vasodilation; sodium nitroprusside

\section{Introduction}

$\mathbf{H}$ UMAN EXPOSURE TO HIGH ALTITUDES is an increasingly common event and as many as 140 million people live at altitudes of $2500 \mathrm{~m}$ or more (Moore, 2001). Industrial and commercial activities at $3500-5000 \mathrm{~m}$ have substantially increased, such as mining activities, which are common in several countries (Jiménez, 1995; Vinnikov et al., 2014). In Chile, over 60,000 workers are intermittently exposed to high altitudes for long periods of time, and this number is expected to surpass 120,000 by 2020 (www.fundacionchile .com/archivos/reporteccm31425187.pdf). Chronic intermittent hypobaric hypoxia $(\mathrm{CIHH})$ constitutes a previously described model of hypoxic exposure (Jalil et al., 1995; Jiménez, 1995; Richalet et al., 2002; Moraga et al., 2014; Vinnikov et al., 2014).

Rats exposed to CIHH failed to gain body weight (BW), presented an increased hematocrit (Hct), right ventricle hypertrophy, systolic hypertension, liver and kidney damage, lower $\alpha$-adrenoceptor density, and upregulated muscarinic cardiac receptors (Germack et al., 2002; Siqués et al., 2006; Brito et al., 2007; Lopez et al., 2009). Furthermore, Siqués et al. (2006) reported that $30 \%$ of rats exposed to CIHH died

Laboratory of Physiology, Hypoxia and Vascular Function, Department of Biomedical Science, Faculty of Medicine, Universidad Católica del Norte, Coquimbo, Chile. 
before the third week; presenting an elevated Hct and BW loss, indicating that a certain population of rats were intolerant. Animals with an increased Hct that did not generate a pathology and/or death were considered tolerant rats; a concept introduced by Lopez et al. (2009). The same authors detected decreased nitric oxide (NO) production during $\mathrm{CIHH}$, at a stimulated altitude of $4600 \mathrm{~m}$. This was due to increased arginase type II activity, suggesting reduced NO availability that correlated with systemic arterial hypertension (AHT) and an increased Hct in intolerant rats. Another mechanism that reduces NO bioavailability is an increased concentration of free radicals, such as the superoxide anion $\left(\mathrm{O}_{2}{ }^{-}\right)$. In fact, rats exposed to $\mathrm{CIHH}$ displayed increased $\mathrm{O}_{2}{ }^{-}$ and decreased NO (Siqués et al., 2014; Brito et al., 2015), supporting the adventitia's role in $\mathrm{O}_{2}{ }^{-}$production. However, vascular function and its link with NO levels in animals exposed to $\mathrm{CIHH}$ have not been studied. Our aim was to measure nitrate and nitrite (NOx) plasma levels and vascular reactivity in response to potassium chloride $(\mathrm{KCl})$, acetylcholine (Ach), and sodium nitroprusside (SNP) in tolerant and intolerant rats exposed to $\mathrm{CIHH}$, in an ex vivo condition using a bath chamber.

\section{Materials and Methods}

Young male Wistar rats (age: 3.0 0.4 months; weight: $250 \pm 25 \mathrm{~g} ; n=30$ ) were randomly divided into two groups: CIHH $2 \times 2$ group ( 2 days of hypoxia followed by 2 days of normoxia, $n=20$ ), and a control group (normoxia, $n=10$ ), during 1 month.

CIHH was performed in a hypobaric chamber at 428 Torr, (equivalent to an altitude of $4600 \mathrm{~m}$ ). This hypobaric condition was maintained by a vacuum bomb with an internal room air flow of $13.4 \mathrm{~L} / \mathrm{min}$ (representing a flow mean of $0.438 \mathrm{~L} /$ min of air per rat) at $22 \pm 2{ }^{\circ} \mathrm{C}$ in a 12 -hour light-dark cycle. Rats were kept in the hypobaric chamber intermittently, 2 days inside the chamber (hypoxic conditions), followed by 2 days outside the chamber (normoxia $\mathrm{CIHH} 2 \times 2$ ), for a period of 1 month, as previously described by Siqués et al. (2006) and Lopez et al. (2009).

The control group was placed in the same room under the same conditions with the exception of hypoxia. Four animals were housed per cage and were provided with food (10 g/day of pellet per rat) and water using current dispensers.

We measured weight, systolic blood pressure (SBP; $\mathrm{mmHg}$ ), and heart rate in rats at basal conditions, every 4 days during the $\mathrm{CIHH}$ period and immediately after they were removed from the hypobaric chamber. BW was measured (Acculab V-1200) 3 hours after leaving the chamber to allow for hydration. To measure blood pressure and heart rate, an inflatable tail-cuff and pressure sensor (RTBP1003220; Kent Scientific, Torrington, CT) were used as previously described by Jhons et al. (1996). The signal was transmitted through a preamplifier to a workbench data-acquisition system, RTBP-001DS worksheet (Kent Scientific), and the average value of six consecutive measurements was calculated. The measurements were performed by placing the animals inside a movement-limiting Plexiglas chamber for 10-20 minutes at a temperature of $22 \pm 0.5^{\circ} \mathrm{C}$.

Rats were handled in accordance with the Guidelines for Humanitarian Treatment of Laboratory Animals. Animals were sacrificed using anesthesia and this protocol was approved by the Ethics Committee of the Medical Faculty of the Universidad Católica del Norte, Coquimbo, Chile.

\section{CIHH tolerance criteria}

Tolerance was evaluated in Wistar rats exposed to $\mathrm{CIHH}$ according to the predictive factors previously described by Lopez et al. (2009).

Tolerant. Tolerant rats were identified as those that presented increased BW and an Hct $<20 \%$, with a maintained SBP.

Intolerant. Intolerant rats were identified as those with a BW loss $>30 \%$, an increased Hct, and an increased SBP $>20 \%$.

\section{Ex vivo small pulmonary arteries}

Animals were anesthetized with sodium thiopentone (100 mg/kg i.p.) and killed by exanguination. The lungs were removed by dissection and immediately immersed in cold saline, and small pulmonary arteries (SPA) (fifth branch arteries $<300 \mu \mathrm{m}$ ) were cleaned carefully, dissected, isolated, and placed in ice-cold saline. For isometric force measurements, arterial segments of $\sim 2 \mathrm{~mm}$ were mounted in a four-channel small vessel myograph (610 M, Multimyograph; Danish Myotechnology, Aarhus, Denmark) and maintained at $37^{\circ} \mathrm{C}$ aerated with $95 \% \mathrm{O}_{2}{ }^{-} 5 \% \mathrm{CO}_{2}$ in Krebs Ringer buffer (KRB). Following an incubation period of 1 hour, the optimal diameter of each artery was calculated by measuring the maximal contractile response to KRB with equimolar replacement of $\mathrm{Na}^{+}$ with $125 \mathrm{mM} \mathrm{K}^{+}$(K-KRB).

Following an equilibration period of at least 30 minutes, concentration-response curves (CRCs) were performed for $\mathrm{KCl}$ (5.6-125 mM), and vasorelaxing curves were performed in the presence of the cholinergic agonist Ach or SNP, a donor of NO. Arteries were previously contracted with $62.5 \mathrm{mM} \mathrm{KCl}$, and increased concentrations of Ach or SNP were added to the bath $\left(10^{-10}\right.$ to $\left.10^{-3} \mathrm{~mol} / \mathrm{L}\right)$. Between each experiment, the arterial preparations were left to return to resting basal tension for at least 30-60 minutes.

The KRB contained (in $\mathrm{mM}$ ) NaCl 118.5, $\mathrm{NaHCO}_{3} 25, \mathrm{KCl}$ 4.7, $\mathrm{KH}_{2} \mathrm{PO}_{4} 1.2, \mathrm{MgSO}_{4} 1.2, \mathrm{CaCl}_{2} 2.5$, and glucose 5.5 with a $\mathrm{pH}$ of 7.4. For the potassium-KRB solution $\left(125 \mathrm{mM} \mathrm{K}^{+}\right)$, all $\mathrm{NaCl}$ was replaced by an equimolar amount of $\mathrm{KCl}$. All chemicals were reagent grade and purchased from Sigma Chemical (St Louis, MO). Ach and SNP were purchased from Sigma Chemical.

\section{Hct and NOx measurements}

Tail blood samples were taken from all animals before the start of the protocol in control or $\mathrm{CIHH}$ groups, and at the end of the protocol.

Hct measurements were obtained in duplicate using a microhematocrit centrifuge (Hawksley, Lancing, UK), and total plasma NOx content was measured using a kit based on the Griess reaction (Cayman Chemical, Ann Arbor, MI). The kit enabled the detection of the sum of NOx by providing an index of NO content. NOx concentrations were reported as $(\mu \mathrm{mol} / \mathrm{L})$.

\section{Statistical analysis}

CRCs were analyzed in terms of potency and maximal responses by fitting experimental data to a sigmoidal equation (Prism 5.0; GraphPad Software, La Jolla, CA). The $\mathrm{KCl}$ contractile response was expressed as tension $(\mathrm{N} / \mathrm{m})$, and the relaxation response to Ach and SNP was expressed as the 
percentage of the $\mathrm{KCl}$ response $\left(\% \mathrm{~K}^{+} 62.5 \mathrm{mM}\right)$. Potency was calculated as $\mathrm{pEC}_{50}$, where $\mathrm{pEC}_{50}=-\log \left[\mathrm{EC}_{50}\right], \mathrm{EC}_{50}$ being the concentration at which $50 \%$ of the maximal response was obtained.

The obtained values are expressed as the mean \pm standard error of the mean. For in vivo studies, groups were compared by two-way analysis of variance (ANOVA) and the post hoc Newman-Keuls test. For ex vivo studies were analyzed using a one-way ANOVA followed by Tukey's multiple comparison test. The Pearson correlation test was used to evaluate the correlation between NOx and SBP, Hct, potassium maximum contractile response $\left(\mathrm{R}_{\mathrm{MAX}}\right)$, Ach maximum dilation ( $\left.\mathrm{D}_{\mathrm{MAX}}\right)$, and SNP D $\mathrm{D}_{\mathrm{MAX}}$. Differences were considered statistically significant when $p<0.05$. (Prism 4.0; GraphPad).

\section{Results}

Using the criteria described previously, 20 rats exposed to $\mathrm{CIHH} 70 \%(14 / 20)$ were identified as tolerant, and $30 \%$ $(6 / 20)$ were identified as intolerant (Fig. 1). Heart rate was neither modified in control nor in CIHH groups.

\section{Ex vivo $S P A$}

Potassium response. The contractile response mediated by $\mathrm{KCl}$ was evaluated in the SPA of rats exposed to $\mathrm{CIHH}$ and a control group. This indirectly measures the muscularization of the SPA used in the study based on their $\mathrm{R}_{\mathrm{MAX}}$ to $\mathrm{KCl}$ (Pearce et al., 1991; Wagerle et al., 1995; Anwar et al., 2001). Tolerant and intolerant rats had a higher vasoconstrictor response to potassium compared with the control group (Fig. 2; Table 1). Furthermore, the intolerant group had an increased $\mathrm{R}_{\text {MAX }}$ compared with the tolerant group. Rats exposed to $\mathrm{CIHH}$ (tolerant and intolerant) presented an increased potency (or $\mathrm{EC}_{50}$ ) compared with the control group $(p<0.05)$.

Ach response. We evaluated the vasodilation effect of Ach in the SPA of rats exposed to CIHH and the control group. Ach evoked minor maximal relaxation in tolerant and intolerant rats exposed to $\mathrm{CIHH}$ compared with the control group (Fig. 3A; Table 1), however, no difference was observed between the tolerant and intolerant group. The $\mathrm{pEC}_{50}$ of the pulmonary vessels in response to Ach was significantly greater in the SPA of the control group compared with rats exposed to CIHH (Fig. 3A; Table 1). No difference was observed between the tolerant and intolerant groups.

SNP response. We evaluated the vasodilation effect of SNP in the SPA of rats exposed to CIHH and the control group. The maximal relaxation response of pulmonary arteries to SNP was lower in tolerant and nontolerant rats exposed to CIHH compared with control rats (Fig. 3B; Table 1). The maximal SNP relaxation response of intolerant rats was also lower compared with tolerant rats. Moreover, the $\mathrm{pEC}_{50}$ of the SPA in response to SNP was significantly increased in rats exposed to CIHH compared with the control group (Fig. 3B; Table 1). However, no difference was observed between the tolerant and intolerant group in the $\mathrm{pEC}_{50}$ for SNP.

Plasma NOx. We studied the effect of CIHH on NOx plasma concentration in tolerant, intolerant, and control rats. We observed a significant decrease in NOx plasma concentration in intolerant rats after exposure to CIHH (Fig. 4). In
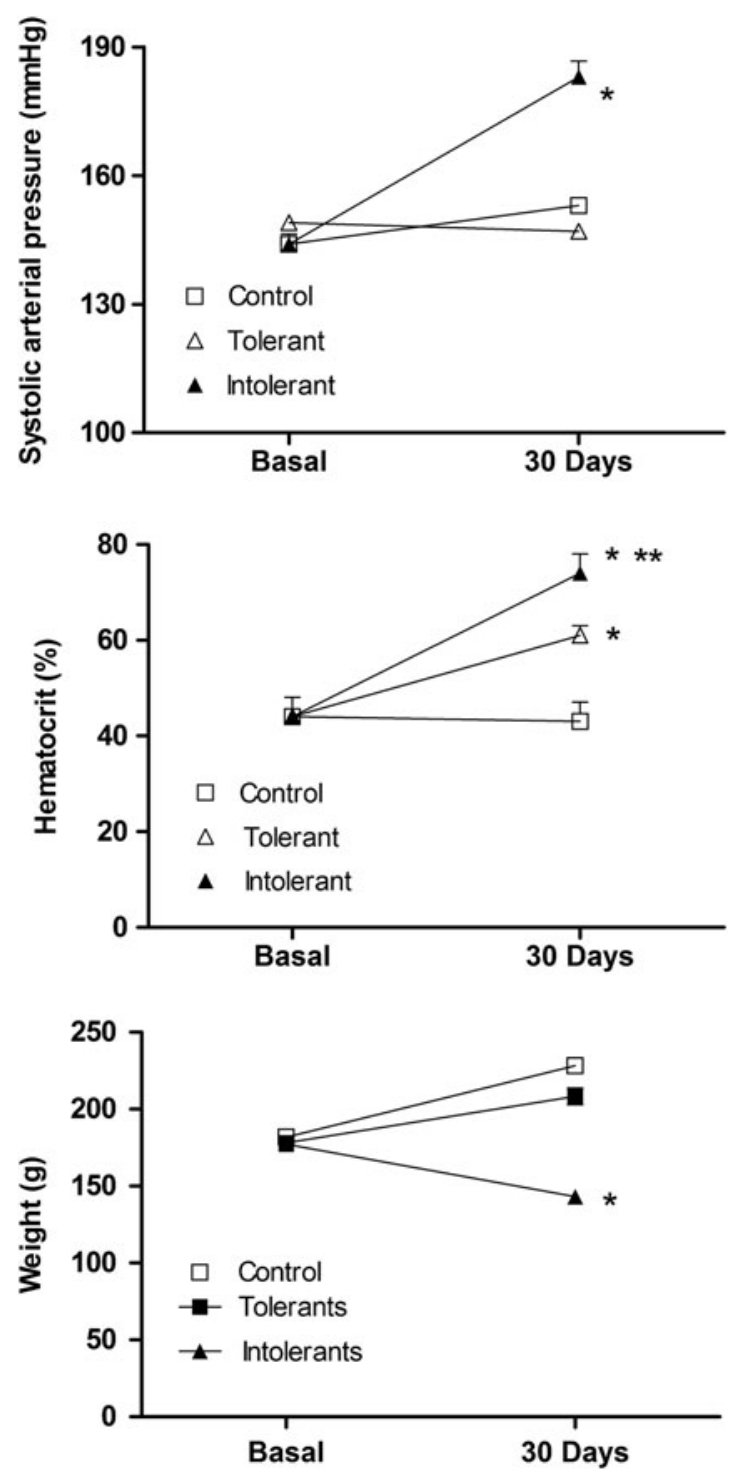

FIG. 1. Cardiovascular and hematological response of rats exposed to CIHH. Upper graph represents a systolic arterial pressure ( $\mathrm{mmHg})$, middle graph represents hematocrit $(\%)$, and lower graph represents weight $(\mathrm{g})$, where: open square (control rats), open triangle (tolerant rats), and close triangle (intolerant rats). Values are means \pm SEM, ${ }^{*} p<0.01$, control vs. intolerant or tolerant and $* * p<0.05$ tolerant vs. intolerant. $\mathrm{CIHH}$, chronic intermittent hypobaric hypoxia; SEM, standard error of the mean.

contrast, NOx concentration in tolerant and control rats was not altered (Fig. 4). No correlations were observed between NOx levels and the variables studied.

\section{Discussion}

There are several types of human exposure to high altitudes. The first corresponds to acute exposure; tourists, climbers, hikers, and so on (Ward et al., 1995). The second type of exposure corresponds to prolonged exposure; people who live at high altitudes $(3000-4500 \mathrm{~m})$ which has been extensively studied in Tibetans, Aymara, and Ethiopian populations during different lengths of time (Beall, 2006); The third type of exposure is chronic intermittent exposure, 


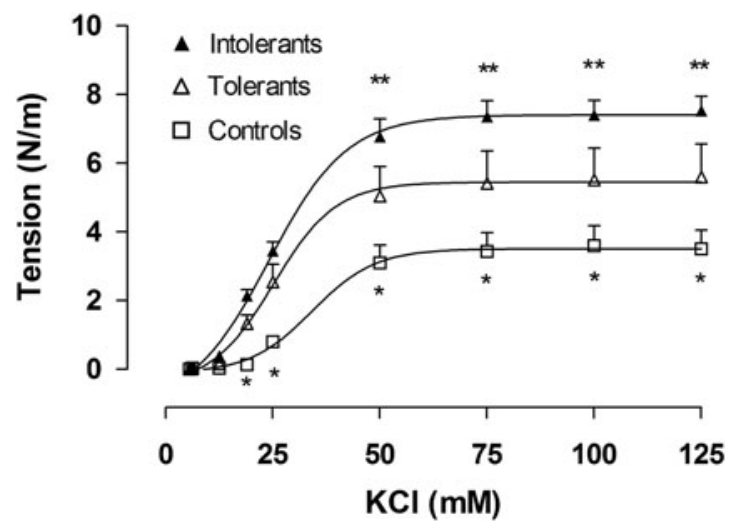

FIG. 2. Concentration-response curve to $\mathrm{KCl}$ in pulmonary small arteries from rats exposed to $\mathrm{CIHH}$ where: open square (control rats), open triangle (tolerant rats), and close triangle (intolerant rats). Values are shown as mean \pm SEM, $* p<0.01$, control vs. intolerant or tolerant and $* * p<0.05$ tolerant vs. intolerant. $\mathrm{KCl}$, potassium chloride.

which occurs in populations that work at high altitudes (Richalet et al., 2002; Moraga et al., 2014). However, information on intermittent exposure to hypobaric conditions and its effect on vascular reactivity in rats is scarce or absent (Thomas and Wanstall, 2003). Studies in animal models have only analyzed histological and molecular changes of vascular pulmonary functions. Our results show that in the $\mathrm{CIHH}$ model, intolerant rats had lower NOx levels, a higher $\mathrm{R}_{\mathrm{MAX}}$ in response to $\mathrm{KCl}$, and a lower dilatator response to SNP compared with tolerant and control rats.

\section{$\mathrm{ClHH}$ and the vasoconstriction response to $\mathrm{KCl}$ in SPA}

The maximum potassium response is higher in the SPA of intolerant rats compared with tolerant and control rats. In addition, higher potassium potency was observed in both intolerant and tolerant rats.

The higher potassium $\mathrm{R}_{\mathrm{MAX}}$ values in intolerant rats could be explained by increased muscularization of the SPA. Pulmonary circulation is sensitive to the lack of oxygen, which promotes hypoxia pulmonary vasoconstriction and pathological remodeling which is characterized by pulmonary artery wall thickening (Makino et al., 2011). Studies have reported a direct relationship between $\mathrm{R}_{\mathrm{MAX}}$ and femoral artery muscle mass (Anwar et al., 2001) and a positive correlation between the maximum potassium response and vascular wall thickness (Pearce et al., 1991; Wagerle et al., 1995). Also, unpublished studies from our laboratory showed that rats exposed to chronic and intermittent normobaric hypoxia, during 1 month at $3500 \mathrm{~m}$, presented vascular wall thickening of the main pulmonary artery which was related to the potassium

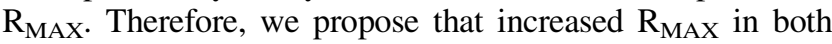
intolerant and tolerant rats is explained by increased muscularization of the medial layer in pulmonary arteries.

Pulmonary vascular remodeling is finely regulated by physical and chemical signals, released by the endothelium and/or adventitia. Among these trophic factors, NO is a vasodilator with an antiproliferative role, meanwhile endothelin, a vasoconstrictor, has a proliferative effect on smooth muscle vasculature; explaining why pulmonary vascular remodeling could be the result of an imbalance between these mediators. Acute hypoxia increases endothelin
Table 1. Summary of the Parameters Obtained FROM THE CONCENTRATION-RESPONSE CURVE OF CONTROL AND CHRONIC INTERMITTENT HYPOBARIC HYPOXIA RATS

\begin{tabular}{|c|c|c|c|}
\hline & Control & Tolerant & Intolerant \\
\hline \multicolumn{4}{|l|}{ CRC-K ${ }^{+}$} \\
\hline $\mathrm{EC}_{50}(\mathrm{mM})$ & $34.2 \pm 1.4$ & $25.2 \pm 1.0^{*}$ & $24.1 \pm 1.4 *$ \\
\hline $\mathrm{R}_{\text {MAX }}(\mathrm{N} / \mathrm{m})$ & $3.51 \pm 0.06$ & $5.45 \pm 0.10 *$ & $7.40 \pm 0.13^{*, \dagger}$ \\
\hline \multicolumn{4}{|l|}{ CRC-Ach } \\
\hline $\mathrm{pEC}_{50}(\mathrm{mM})$ & $6.071 \pm 0.004$ & $4.815 \pm 0.187 *$ & $4.242 \pm 0.353^{*}$ \\
\hline $\begin{array}{l}\text { Relaxation max } \\
\left(\% \mathrm{~K}^{+} 62.5\right)\end{array}$ & $46.9 \pm 0.6$ & $28.7 \pm 2.1^{*}$ & $29.9 \pm 1.8^{*}$ \\
\hline \multicolumn{4}{|l|}{ CRC-SNP } \\
\hline pEC50 (mM) & $6.329 \pm 0.1$ & $7.010 \pm 0.270 *$ & $7.218 \pm 0.170 *$ \\
\hline $\begin{array}{l}\text { Relaxation max } \\
\left(\% \mathrm{~K}^{+} 62.5\right)\end{array}$ & $89.0 \pm 1.5$ & $78.5 \pm 1.1 *$ & $72.4 \pm 2.8^{*} \dagger$ \\
\hline
\end{tabular}

Mean \pm standard error of the mean.

*Control vs. tolerant and intolerant.

${ }^{\dagger}$ Tolerant vs. intolerant $(p<0.05)$.

Ach, acetylcholine; CRC, concentration-response curves; $\mathrm{R}_{\mathrm{MAX}}$, maximum contractile response; SNP, sodium nitroprusside.

expression and reduces NO expression (Gao and Raj, 2010). However, chronic hypoxia increased NO production and reduced soluble guanylyl cyclase (sGC) expression (Herrera et al., 2008a). An additional possibility is that $\mathrm{CH}$ increases the sensitivity of the contractile apparatus to $\mathrm{Ca}^{+2}$ and mediates this response via activation of the RhoA/Rho kinase (ROK) pathway, and consequently inhibits the myosin light-chain phosphatase, a central component of vascular smooth muscle contraction (Jernigan and Resta, 2014). However, our study corroborated decreased NOx levels in intolerant animals during CIHH (Siqués et al., 2014; Brito et al., 2015), which could partially explain the increased potassium $\mathrm{R}_{\mathrm{MAX}}$.

Increased potassium potency, observed in both intolerant and tolerant rats, suggests that $\mathrm{CIHH}$ increases the expression of contractile machinery stimulated by depolarization at lower potassium concentrations. According to the data in Table 1, the modified extracellular potassium concentration influences the electrochemical gradient and, according to the Nernst equation, predicts that the voltage threshold for contraction of the SPA of intolerant and tolerant rats is more negative than the control group (Papamatheakis et al., 2012; Makino et al., 2011; Jernigan and Resta, 2014). This contraction capacity at a lower potassium concentration could be explained by reduced expression of potassium channels in pulmonary arteries (i.e., $\mathrm{Kv}$ $1.2, \mathrm{Kv} 1.5$, and $\mathrm{Kv} 2.1$ ) in rats exposed to chronic hypoxia (Wang et al., 2005; Whitman et al., 2008; and more detail see Sylvester et al., 2012); increased expression of the L and Ttype voltage-dependent calcium channel (Makino et al., 2011; Wan et al., 2013; Jernigan and Resta, 2014); and/or the coupled electro-mechano-contractile machinery present, similar to that described by Nauli et al. (2005) who showed that $\mathrm{Ca}^{+2}$ had an increased capacity for promoting myosin phosphorylation in adult sheep basilar arteries during chronic hypoxia. Similar results were described by Broughton et al. (2010) in the pulmonary arteries of rats exposed to chronic hypoxia. Since the previously mentioned studies were performed in animals submitted to chronic hypoxia, we propose that $\mathrm{CIHH}$ follows the same mechanism. However, pharmacological and molecular studies should be performed to confirm this hypothesis. 
A

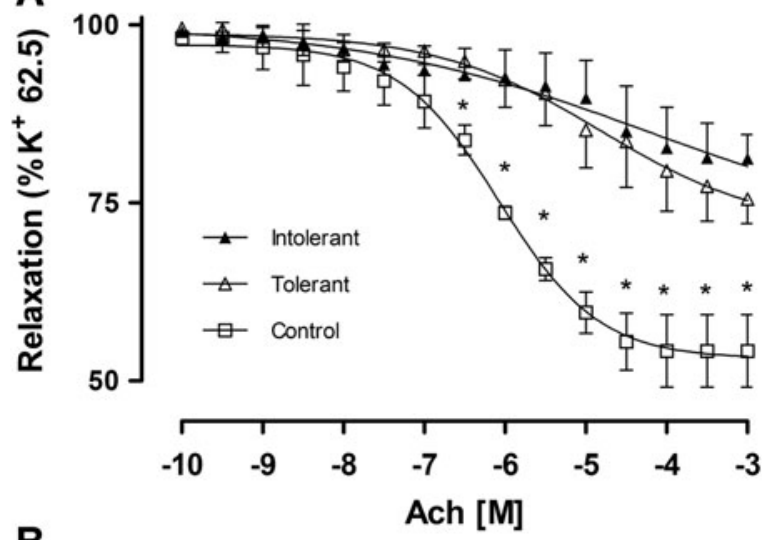

B

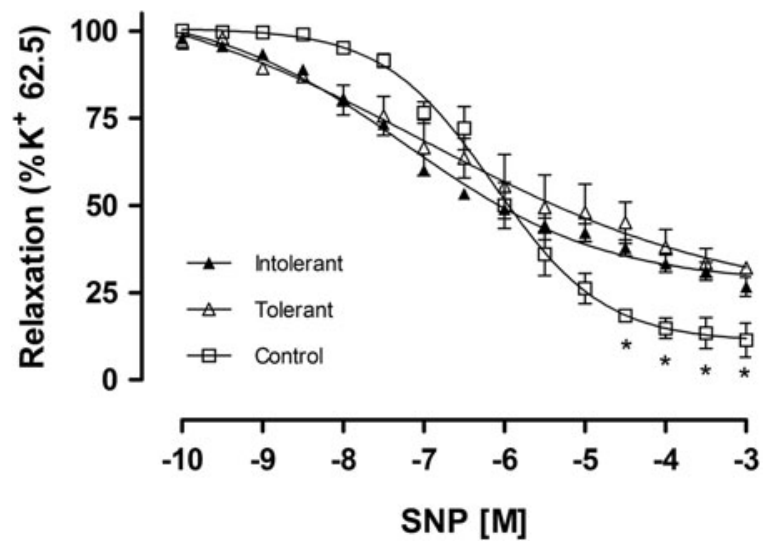

FIG. 3. (A) Responses to Ach in pulmonary small arteries from rats exposed to CIHH. (B) Responses to SNP in pulmonary small arteries from rats exposed to $\mathrm{CIHH}$ where: open square (control rats), open triangle (tolerant rats), and close triangle (intolerant rats). Values are shown as mean \pm SEM, $* p<0.01$, control vs. intolerant or tolerant. Ach, acetylcholine; SNP, sodium nitroprusside.

\section{$\mathrm{ClHH}$ attenuates Ach-induced vasodilation in pulmonary arteries}

In mammalian vascular endothelium, several extracellular signals can stimulate nitric oxide synthase (NOS), increasing NO production; including Ach, shear stress, and so on (Furchgott and Zawadzki, 1980). The NO produced by endothelium rapidly diffuses into smooth muscle cells where it activates sGC, generating cyclic guanosine monophosphate (cGMP), which mediates vasodilation (Moncada et al., 1991). In normal conditions, $\mathrm{NO}$ production plays a key role in the physiological regulation of the cardiovascular system. In contrast, decreased NO precursors, impaired synthesis, and/or increased degradation could be associated with diseases such as hypertension, pulmonary hypertension, remodeling, atherosclerosis, among others (Varadharaja et al., 2015).

L-arginine is an essential amino acid in mammals and a NO precursor. Therefore, decreased L-arginine availability can contribute to NO deficiency. However, the L-arginine plasma concentration is not altered in rats exposed to $\mathrm{CIHH}$ (Lüneburg et al., 2016). One explanation is that arginase converts L-arginine into ornithine and urea, reducing Larginine availability and NO production. NO production can be regulated by L-arginine plasma levels as a result of the competition between NOS and arginase (Pernow and

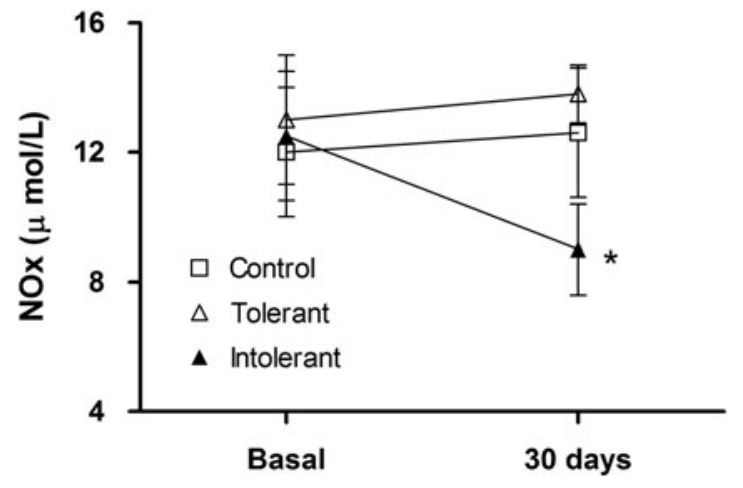

FIG. 4. NOx plasmatic concentration of rats exposed to $\mathrm{CIHH}$. Values are mean \pm SEM, where: open square (control rats), open triangle (tolerant rats), and close triangle (intolerant rats). ${ }^{*} p<0.01$, intolerant vs. tolerant and control. NOx, nitrate and nitrite.

Jung, 2013). We previously reported that exposure to CIHH upregulates arginase II (Lopez et al., 2009) and increases arginase activity (Lüneburg et al., 2016). Therefore, we propose that our intolerant rats present increased arginase II expression, leading to decreased bioavailability for NOS and decreased NO; inducing the development of pulmonary AHT in rats exposed to CIHH. In addition, several mechanisms contribute to endothelial NOS (eNOS) uncoupling, causing endothelial dysfunction. However, nicootinamide adenine dinucleotide phosphatase oxidase plays a crucial role in eNOS uncoupling since it produces reactive oxygen species (Cuzzocrea et al., 2004; Norton et al., 2013). Intolerant rats exposed to $\mathrm{CIHH}$ displayed increased $\mathrm{O}_{2}{ }^{-}$and decreased NO (Siqués et al., 2014; Brito et al., 2015; Lüneburg et al., 2016). In agreement with these studies, our intolerant rats have low NOx levels and a low vascular response to Ach. Another condition that could explain the reduced NO levels in animals exposed to $\mathrm{CIHH}$ is that $\mathrm{NO}$ can undergo a number of reactions under different biological or physiological conditions. Also, NO is scavenged by oxyhemoglobin to yield methemoglobin and inorganic nitrate (Beckman and Koppenol, 1996). In agreement with previous observations, intolerant rats have a higher hemoglobin concentration, decreased NO availability, and/or an increased Hct (viscosity) with increased vascular resistance of the pulmonary and systemic circulatory systems.

\section{CIHH attenuates vasodilation in response to SNP in the pulmonary arteries}

As previously described, a blunted endothelium response to Ach can be induced by decreased NO precursor levels, synthesis, or increased degradation.

To demonstrate that endothelium is altered by $\mathrm{CIHH}$, NOdonor agents (SNP or SNAP) could be used. The use of NOdonor agents activates sGC by an endothelium-independent pathway, increasing cGMP and promoting pulmonary vasodilation by inactivating calcium channels and/or decreasing the sensitivity of the contractile apparatus to calcium (Jernigan and Rest, 2002). In our study, SNP promoted a minor dilation response of $18.7 \%$ and $11.7 \%$ in intolerant and tolerant rats, respectively, compared with control rats. In 
addition, when we analyzed the $\mathrm{R}_{\mathrm{MAX}}$ ratio $\left[\left(\mathrm{R}_{\mathrm{MAX}} \mathrm{SNP}-\right.\right.$ $\mathrm{R}_{\text {MAX }}$ Ach) $\left./ \mathrm{R}_{\text {MAX }} \mathrm{SNP}\right] \times 100$, this represented a recovered vasodilation response in the presence of exogenous NO. We observed that intolerant rats recovered $58.6 \%$ of the dilation response in the presence of SNP, compared with $63.4 \%$ in tolerant rats and $47 \%$ in control rats. Therefore, $\mathrm{CIHH}$ promotes an endothelium dysfunction that decreases the dilation response to Ach, reducing NO availability. This suggests that in the $\mathrm{CIHH}$ pathway $\mathrm{NO} \rightarrow \mathrm{sGC} \rightarrow \mathrm{cGMP} \rightarrow \mathrm{PKG} \rightarrow$ vasodilation is reduced. The results from studies performed in rats exposed to $\mathrm{CIHH}$ show that they develop a right ventricular hypertrophy (Lüneburg et al., 2016) as a result of reduced NO availability rather than reduced sGC activity. However, studies performed in newborns exposed to chronic hypoxia showed increased phosphodiesterase type V (PDE5) activity and/or decreased pulmonary artery sensitivity to cGMP (Jernigan and Resta, 2002). Furthermore, Herrera et al. (2008b) showed decreased sGC expression in newborns exposed to chronic hypoxia. Unfortunately no information about the expression mechanisms in animals exposed to CIHH exists.

\section{Conclusion}

After exposure to $\mathrm{CIHH}, 30 \%$ of intolerant rats had a lower NOx concentration, an increased response to $\mathrm{KCl}$, and a decrease in the small pulmonary artery response to Ach and SNP.

\section{Acknowledgments}

The authors thank Gabriela Lamas and Hervis Galleguillos for their invaluable assistance and collaboration. This work was supported by the FONDECYT 11075096 and 07CN13ISM152 INNOVA CORFO grant, Chile.

\section{Author Disclosure Statement}

No competing financial interests exist.

\section{References}

Anwar MA, Kimberly J, Docherty CC, Poston L, and Nathanielsz PW. (2001). Developmental changes in reactivity of small femoral arteries in the fetal and postnatal baboon. Am J Obstet Gynecol 184:707-712.

Beall CM. (2006). Andean, Tibetan, and Ethiopian patterns of adaptation to high-altitude hypoxia. Integr Comp Biol 46: $18-24$.

Beckman JS, and Koppenol WH. (1996). Nitric oxide, superoxide, and peroxynitrite: The good, the bad, and ugly. Am J Physiol 271:C1424-C1437.

Brito J, Siqués P, León-Velarde F, De La Cruz JJ, López V, and Herruzo R. (2007). Chronic intermittent hypoxia at high altitude exposure for over 12 years: Assessment of hematological, cardiovascular, and renal effects. High Alt Med Biol 8:236-244.

Brito J, Siques P, Arribas SM, López de Pablo AL, González MC, Naveas N, et al. (2015). Adventitial alterations are the main features in pulmonary artery remodeling due to longterm chronic intermittent hypobaric hypoxia in rats. Biomed Res Int 2015:169841.

Broughton BR, Jernigan NL, Norton CE, Walker BR, and Resta TC. (2010). Chronic hypoxia augments depolarizationinduced $\mathrm{Ca}^{2+}$ sensitization in pulmonary vascular smooth muscle through superoxide-dependent stimulation of RhoA. Am J Physiol Lung Cell Mol Physiol 298:L232-L242.
Cuzzocrea S, Mazzon E, Dugo L, Di Paola R, Caputi AP, and Salvemini D. (2004). Superoxide: A key player in hypertension. FASEB J 18:94-101.

Furchgott RF, and Zawadzki JV. (1980). The obligatory role of endothelial cells in the relaxation of arterial smooth muscle by acetylcholine. Nature 288:373.

Gao Y, and Raj JU. (2010). Regulation of the pulmonary circulation in the fetus and newborn. Physiol Rev 90:12911335.

Germack R, Leon-Velarde F, Valdes De La Barra R, Farias J, Soto G, and Richalet JP. (2002). Effect of intermittent hypoxia on cardiovascular function, adrenoceptors and muscarinic receptors in Wistar rats. Exp Physiol 87:453460.

Herrera EA, Reyes RV, Giussani DA, Riquelme RA, Sanhueza EM, Ebensperger G, et al. (2008a). Carbon monoxide: A novel pulmonary artery vasodilator in neonatal llamas of the Andean altiplano. Cardiovasc Res 77:197-201.

Herrera EA, Ebensperger G, Krause BJ, Riquelme RA, Reyes RV, Capetillo M, et al. (2008b). Sildenafil reverses hypoxic pulmonary hypertension in highland and lowland newborn sheep. Pediatr Res 63:169-175.

Jalil J, Casanegra P, Braun S, Chamorro G, Saldías F, Beroíza $\mathrm{T}$, et al. (1995). Working at high altitude in Andean miners from Chile: Human adaptation to long term intermittent hypobaric hypoxia. In: Hypoxia and the Brain. JR Sutton, CS Houston, and G Coates, eds. Queen City Printers, Burlington, VT, pp. 292-297.

Jernigan NL, and Resta TC. (2002). Chronic hypoxia attenuates cGMP-dependent pulmonary vasodilation. Am J Physiol Lung Cell Mol Physiol 282:L1366-L1375.

Jernigan NL, and Resta TC. (2014). Calcium homeostasis and sensitization in pulmonary arterial smooth muscle. Microcirculation 21:259-271.

Jhons C, Gavras I, Handy DE, Salomao A, and Gavras H. (1996). Model of experimental hypertension in mice. Hypertension 28:1064-1069.

Jiménez D. (1995). High altitude intermittent chronic exposure: Andean miners. In: Hypoxia and the Brain. JR Sutton, CS Houston, and G Coates, eds. Queen City Printers, Burlington, VT, pp. 284-291.

Lopez V, Siques P, Brito J, Vallejos C, Naveas N, Carvallo C, et al. (2009). Upregulation of arginase expression and activity in hypertensive rats exposed to chronic intermittent hypobaric hypoxia. High Alt Med Biol 10:373-381.

Lüneburg N, Siques P, Brito J, Arriaza K, Pena E, Klose H, et al. (2016). Long-term chronic intermittent hypobaric hypoxia in rats causes an imbalance in the asymmetric dimethylarginine/nitric oxide pathway and ROS activity: A possible synergistic mechanism for altitude pulmonary hypertension? Pulm Med 2016:6578578.

Makino A, Firth AL, and Yuan JX. (2011). Endothelial and smooth muscle cell ion channels in pulmonary vasoconstriction and vascular remodeling. Compr Physiol 1:1555-1602.

Moncada S, Palmer RMJ, and Higgs EA. (1991). Nitric oxide physiology, pathophysiology and pharmacology. Pharmacol Rev 120:227-237.

Moore LG. (2001). Human genetic adaptation to high altitude. High Alt Med Biol 2:257-279.

Moraga FA, Jiménez D, Richalet JP, Vargas M, and Osorio J. (2014). Periodic breathing and oxygen supplementation in Chilean miners at high altitude $(4200 \mathrm{~m})$. Respir Physiol Neurobiol 203:109-115.

Nauli SM, Williams JM, Gerthoffer WT, and Pearce J. (2005). Chronic hypoxia modulates relations among calcium, myosin 
light chain phosphorylation, and force differently in fetal and adult ovine basilar arteries. J Appl Physiol 99:120-127.

Norton CE, Broughton BR, Jernigan NL, Walker BR, and Resta TC. (2013). Enhanced depolarization-induced pulmonary vasoconstriction following chronic hypoxia requires EGFRdependent activation of $\mathrm{NAD}(\mathrm{P}) \mathrm{H}$ oxidase 2. Antioxid Redox Signal 18:1777-1788.

Papamatheakis DG, Patel JJ, Blood Q, Merritt TT, Longo LD, and Wilson SM. (2012). Depolarization-dependent contraction increase after birth and preservation following long-term hypoxia in sheep pulmonary arteries. Pulm Circ 2:41-53.

Pearce WJ, Hull AD, and Longo LD. (1991). Developmental changes in ovine cerebral artery composition and reactivity. Am J Physiol 258:H458-R465.

Pernow J, and Jung C. (2013). Arginase as a potential target in the treatment of cardiovascular disease: Reversal of arginine steal? Cardiovasc Res 98:334-343.

Richalet JP, Donoso MV, Jiménez D, Antezana AM, Hudson C, Cortès $\mathrm{G}$, et al. (2002). Chilean miners commuting from sea level to $4500 \mathrm{~m}$ : A prospective study. High Alt Med Biol 3: 159-166.

Sylvester JT, Shimoda LA, Aaronson PI, and Ward JPT (2012). Hypoxic pulmonary vasoconstriction. Physiol Rev 92:367520.

Siqués P, Brito J, León-Velarde F, Barrios L, Cruz JJ, López V, et al. (2006). Time course of cardiovascular and hematological responses in rats exposed to chronic intermittent hypobaric hypoxia (4600 m). High Alt Med Biol 7:72-80.

Siqués P, López de Pablo AL, Brito J, Arribas SM, Flores K, Arriaza K, et al. (2014). Nitric oxide and superoxide anion balance in rats exposed to chronic and long term intermittent hypoxia. Biomed Res Int 2014:610474.

Thomas BJ, and Wanstall JC. (2003). Alterations in pulmonary vascular function in rats exposed to intermittent hypoxia. Eur J Pharmacol 477:153-161.

Varadharaja S, Porter K, Pleister A, Wannemacher J, Sow A, Jarjoura D, et al. (2015). Endothelial nitric oxide synthase uncoupling: A novel pathway in OSA induced vascular endothelial dysfunction. Respir Physiol Neurobiol 207:40-47.
Vinnikov D, Brimkulov N, Krasotski V, Redding-Jones R, and Blanc PD. (2014). Risk factors for occupational acute mountain sickness. Occup Med (Lond) 64:483-489.

Wagerle LC, Moliken W, and Russo P (1995). Nitric oxide and $\beta$-adrenergic mechanisms modify contractile responses to norepinephrine in ovine fetal and newborn cerebral arteries. Pediatr Res 38:237-242.

Wan J, Yamamura A, Zimnicka AM, Voiriot G, Smith KA, Tang H, et al. (2013). Chronic hypoxia selectively enhances $\mathrm{L}$ - and $\mathrm{T}$-type voltage-dependent $\mathrm{Ca} 2+$ channel activity in pulmonary artery by upregulating Cav1.2 and Cav3.2. Am J Physiol Lung Cell Mol Physiol 305:L154-L164.

Wang J, Weigand L, Wanq W, Sylvester JT, and Shimoda LA. (2005). Chronic hypoxia inhibits Kv channel gene expression in rat distal pulmonary artery. Am J Physiol Lung Cell Mol Physiol 288:L1049-L1058.

Ward MP, Milledge JS, and West JB. (1995). Acute and subacute mountain sickness. In: High Altitude Medicine and Physiology. Chapman \& Hall Medical, London, UK, pp. 366-387.

Whitman EM, Pisarcik S, Luke T, Fallon M, Wang J, Sylvester JT, Semenza GL, and Shimoda LA. (2008). Endothelin-1 mediates hypoxia induced inhibition of voltage-gated $\mathrm{K}$ channel expression in pulmonary arterial myocytes. Am J Physiol Lung Cell Mol Physiol 294:L309-L318.

Address correspondence to: Fernando A. Moraga, BSc, MSc, PhD Laboratory of Physiology, Hypoxia and Vascular Function

Department of Biomedical Science Faculty of Medicine

Universidad Católica del Norte Larrondo 1281, PO-Box 117 Coquimbo Chile

E-mail: fmoraga@ucn.cl

Received January 17, 2017; accepted in final form January 3, 2018. 